\title{
Young infants with severe tetralogy of Fallot: Early primary surgery versus transcatheter palliation
}

\author{
Travis J. Wilder, MD, ${ }^{\mathrm{a}}$ Glen S. Van Arsdell, MD, ${ }^{\mathrm{a}, \mathrm{d}}$ Lee Benson, MD, ${ }^{\mathrm{b}, \mathrm{c}}$ Eric Pham-Hung, BSc, ${ }^{\mathrm{a}}$ \\ Michael Gritti, BSc, ${ }^{a}$ Alexandra Page, BSc, ${ }^{\mathrm{a}}$ Christopher A. Caldarone, MD, ${ }^{\mathrm{a}, \mathrm{d}}$ and Edward J. Hickey, MD ${ }^{\mathrm{a}, \mathrm{d}}$
}

\section{ABSTRACT}

Background: Infants with severe tetralogy of Fallot may undergo (1) early primary surgical repair (EARLY) or (2) early transcatheter palliation (CATH) before delayed surgical repair. We compared these strategies with (3) elective singlestage tetralogy of Fallot repair (IDEAL).

Methods: From 2000 to 2012, 453 children underwent tetralogy of Fallot repair (excluding systemic-pulmonary shunts), including 383 in the IDEAL (75\%), 42 in the EARLY (9\%), and 28 in the CATH (6\%) groups. IDEAL repair at The Hospital for Sick Children occurs after 3 months. Risk-adjusted hazard analysis compared freedom from surgical or catheter reintervention. Somatic size, branch pulmonary artery size, and right ventricle systolic pressure were modeled using 2780 echocardiogram reports via mixed-model regression.

Results: CATH involved right ventricular outflow tract stent in 18 patients, right ventricular outflow tract balloon in 9 patients, and ductal-stent in 1 patient. Three patients died (1 per group). Risk-adjusted freedom from surgical reoperation was $89 \% \pm 4 \%, 88 \% \pm 5 \%$, and $85 \% \pm 6 \%$ for the IDEAL, EARLY, and CATH groups, respectively, at 10 years. Patients in the EARLY and CATH groups had similar reoperation rates, except for neonates $(<1$ month), for whom EARLY repair conferred an increased risk of reoperation. Risk-adjusted freedom from catheter reintervention was lower in the EARLY group (76\%) and especially for the CATH group (53\%) at 10 years versus the IDEAL group $(83 \%)$. Somatic growth and progression of right ventricle systolic pressure were similar among groups at 8 years. Although those undergoing EARLY $(P=.02)$ and CATH $(P=.09)$ tend to have smaller branch pulmonary arteries initially, late pulmonary artery size was not significantly different among groups.

Conclusions: Early primary repair for neonates may increase surgical reoperation, whereas transcatheter palliation comes at a cost of increased catheter reintervention. However, overall outcomes between groups, in terms of survival, growth, and hemodynamic parameters, were comparable, suggesting that both strategies are a reasonable option for children with severe tetralogy of Fallot. (J Thorac Cardiovasc Surg 2017;154:1692-1700)

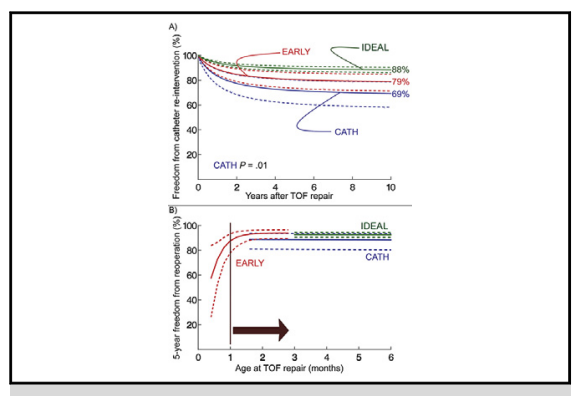

Risk-adjusted freedom from any catheter reintervention stratified by timing of repair.

\section{Central Message}

Despite being associated with more frequent invasive reinterventions, early primary repair and early transcatheter palliation are effective strategies for the treatment of infants with severe TOF

\section{Perspective}

Infants with severe TOF who require early intervention can undergo primary surgical repair or transcatheter palliation with delayed elective repair. Although early intervention, either surgical or transcatheter, comes at a cost of increased invasive reinterventions versus ideal elective repair after 3 months of age, outcomes in terms of survival, somatic growth, branch PA growth, and RVSP are comparable.

See Editorial Commentary page 1701.

See Editorial page 1690.
From the a Division of Cardiovascular Surgery, Department of Surgery, and ${ }^{\mathrm{b}}$ Division of Cardiology, Department of Pediatrics, The Hospital for Sick Children; and ${ }^{\mathrm{c}} \mathrm{Di}$ vision of Cardiology, Department of Medicine, and ${ }^{\mathrm{d}}$ Division of Cardiovascular Surgery, Department of Surgery, University of Toronto, Toronto, Ontario, Canada. An abstract incorporating part of these data was presented at the American Heart Association Scientific Session, Orlando, Florida, November 7-11, 2015.

Received for publication Jan 19, 2016; revisions received April 11, 2017; accepted for publication May 9, 2017; available ahead of print June 27, 2017

Address for reprints: Edward J. Hickey, MD, The Hospital for Sick Children, Room 1518B, 555 University Ave, Toronto, Ontario M5G 1X8, Canada (E-mail: edward. hickey@sickkids.ca).

0022-5223/\$36.00

Copyright (c) 2017 by The American Association for Thoracic Surgery

http://dx.doi.org/10.1016/j.jtcvs.2017.05.042
The general consensus for stable children with tetralogy of Fallot (TOF) is to perform an elective repair after early infancy, but before 1 year of age, ideally between approximately 6 and 12 months of age. ${ }^{1}$ However, the optimal

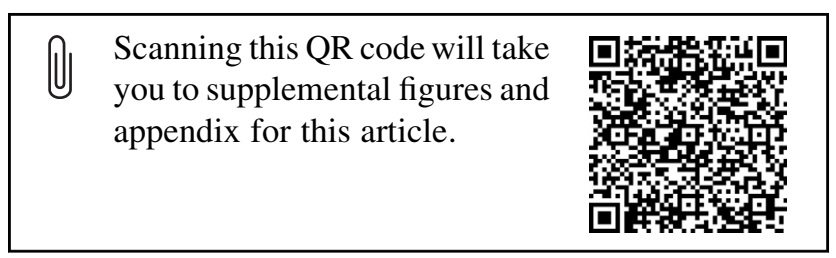



Abbreviations and Acronyms
CATH = early transcatheter palliation
EARLY $=$ early primary surgical repair
IDEAL $=$ elective single-stage tetralogy of Fallot repair
$\begin{array}{ll}\mathrm{PA} & =\text { pulmonary artery } \\ \mathrm{PE} & =\text { parameter estimate }\end{array}$
PI $\quad=$ pulmonary valve insufficiency
RVSP $=$ right ventricular systolic pressure
SPS = systemic-pulmonary shunt
TOF $=$ tetralogy of Fallot

type and timing of initial palliation for neonates and young infants who are severely symptomatic remain unclear. ${ }^{2}$ One strategy is to perform an initial systemic-pulmonary shunt (SPS) followed by formal repair (2 stage). Although this remains a successful strategy among many institutions, ${ }^{3-5}$ in the mid-1990s, The Hospital for Sick Children abandoned initial SPS as a palliative strategy. Instead, if a child presents with cyanosis or becomes progressively cyanotic before 3 months of age, alternative treatment strategies include (1) performing an early, complete primary repair or (2) performing early transcatheter palliation followed by elective surgical repair after 3 months of age.

Survival for patients after TOF repair during the contemporary era is excellent, with early survival among some centers reportedly close to $100 \%$, irrespective of initial strategy. ${ }^{3,5,6}$ Although previous reports compared outcomes between early shunting versus primary repair for young, symptomatic children with $\mathrm{TOF}^{2,3,5}$ there are limited data on the efficacy of early transcatheter palliation for cyanotic infants. Furthermore, despite established reports of excellent survival after early primary repair, data comparing strategies for early intervention, in terms of morbidly, growth, and hemodynamic parameters, are incomplete. Therefore, we compared outcomes between early primary surgical repair and early transcatheter intervention. Patients who underwent an elective "ideal" primary repair after 3 months of age were included as a reference comparison.

\section{PATIENTS AND METHODS}

\section{Study Cohort and Analysis Strategy}

At The Hospital for Sick Children from 2000 to 2012, 453 children with typical TOF morphology (mean overall follow-up, $5.1 \pm 4.1$ years) underwent surgical repair without SPS palliation, forming the analysis cohort for this study. Children with major aortopulmonary collateral artery-dependent pulmonary blood flow were excluded. Since the late 1990s, The Hospital for Sick Children has essentially abandoned SPS procedures as a palliative strategy for children with TOF. When possible, The Hospital for Sick Children favors single-staged, elective primary repair between 3 and 9 months of age (elective single-stage TOF repair [IDEAL]). However, for children who present with acute cyanotic episodes or become progressively cyanotic while awaiting definitive repair, our approach has traditionally been to perform a complete early repair before 3 months of age (early primary surgical repair [EARLY]). Alternatively, especially when the risk profile for surgery is unfavorable, The Hospital for Sick Children has increasingly adopted a strategy of early transcatheter palliation before 3 months, followed by elective repair during the usual time frame (early transcatheter palliation $[\mathrm{CATH}]$ ).

We compared the outcomes between the EARLY and CATH groups, incorporating children who underwent an IDEAL repair as a control. The overall mortality in this series was low (3 total deaths), with 1 death occurring in each group. Survival was not selected as a primary end point because the limited number of deaths precludes a formal statistical analysis. Therefore, efficacy between EARLY and CATH strategies, referenced to IDEAL repair, was compared by evaluating the freedom from surgical reoperation, all catheter reinterventions, and catheter reinterventions directed at the branch pulmonary arteries (PAs). In addition, postrepair somatic growth, branch PA growth, right ventricular systolic pressure, and pulmonary valve insufficiency (PI) were characterized using longitudinal follow-up data via mixed-model regression.

\section{Analysis and Presentation}

The study was approved by our institutional research ethics board. Individual patient consent was not required because patients were not contacted directly. A thorough retrospective review of medical records was conducted. Preoperative, intraoperative, postoperative, and follow-up data were obtained from clinical reports. Variables were extracted and processed from these records as previously described. ${ }^{7}$ Operative, demographic, diagnostic, and interventional information up to the primary surgical repair were included as baseline variables. Univariate and multivariable testing of baseline variables were undertaken for risk hazard and longitudinal regression analyses. For details on variable selection, see Appendix E1.

For descriptive statistics, statistical comparisons were made only between EARLY and CATH groups (statistics for the IDEAL group were summarized as a reference). Continuous variables were compared between EARLY and CATH groups with the Kruskal-Wallis test using Wilcoxon rank scores. The frequencies for categoric variables were compared using a chi-square test of independence or Fisher exact test. Data are presented as median with range, mean \pm standard deviation, or frequency. For hazard and longitudinal analyses, variables for CATH and EARLY were entered into each regression model (whether significant or not), thus allowing their statistical significance to be compared with the reference IDEAL group. Analyses were performed with SAS statistical software (version 9.2; SAS Institute, Inc, Cary, NC).

Freedom from surgical reoperation and catheter reintervention. The freedom from surgical reoperation and catheter reintervention was evaluated via parametric multiphase hazard models constructed on the basis of underlying Kaplan-Meier estimates of time to event. ${ }^{8}$ These models capture individual phases of risk, each of which can independently be subjected to risk-hazard (multivariable) regression analysis and represented as the survival function (for additional details, see http://www.lerner.ccf.org/qhs/software/hazard/). ${ }^{8}$

Baseline parametric models assessing the rate of transition (hazard function) from date of primary TOF repair to each event (surgical reoperation or catheter reintervention) were created. Subsequently, univariate and multivariable analyses were conducted, incorporating baseline characteristics as risk factors. Final variable selection for risk factors was guided by bootstrap resampling ( $\mathrm{n}=500$, threshold for selection $P=.10)$ (Appendix E1).

Longitudinal analysis of repeated echocardiograms. In total, 2780 post-TOF repair echocardiogram reports were reviewed for all 453 patients (Figure E1). At 10 years postrepair, 9\% of children $(\mathrm{n}=40)$ had available echocardiogram data. This allowed temporal trends to be evaluated up to 8 years after TOF repair, such that more than $10 \%$ of the population had available data at last follow-up.

The baseline, postoperative trends for weight (kilograms), branch PA size (millimeters), right ventricular systolic pressure (RVSP) (millimeters of mercury), and the prevalence of PI initially were characterized via nonlinear 
mixed-model regression analysis (SAS PROC NLMIXED). ${ }^{10-12}$ The presence of moderate or worse (moderate or more) PI was considered clinically important insufficiency. Therefore, insufficiency is expressed as the prevalence of PI over time. Subsequently, in a manner similar to standard regression, univariate and multivariable analyses were conducted using baseline variables to identify important factors associated with each postoperative parameter. ${ }^{13}$ See Appendix E1 for further details.

\section{RESULTS}

\section{Patient Characteristics}

Of all 453 patients, $383(75 \%)$ underwent primary TOF repair beyond 3 months of age (IDEAL), 42 (9\%) underwent EARLY primary repair at 3 months or less, and $28(6 \%)$ underwent palliative CATH intervention before TOF repair (Table 1). CATH intervention included 18 RVOT stents, 9 RVOT balloon dilations, and 1 ductal stent placement, and were successful in delaying definitive repair in all but 2 patients who underwent TOF repair at 1.5 months and 2 months. Among all 453 patients there were 3 total deaths, with 1 death in each group. As such, overall mortality was not significantly different between the EARLY and CATH groups.

TABLE 1. Characteristics for children with tetralogy of Fallot stratified by timing of intervention

\begin{tabular}{|c|c|c|c|c|}
\hline Variable & $\frac{\text { IDEAL }(\mathrm{N}=383)}{\text { Median }(\text { range }) \text { or } \mathbf{N}(\%)}$ & $\frac{\text { EARLY }(\mathrm{N}=42)}{\text { Median }(\text { range }) \text { or } N(\%)}$ & $\frac{\text { CATH }(\mathrm{N}=28)}{\text { Median (range) or } \mathbf{N}(\%)}$ & $P^{*}$ \\
\hline Age at first intervention (d) & $189(92-5527)$ & $50(9-90)$ & $13(1-306)$ & $<.001$ \\
\hline Age at primary repair (mo) & $6.3(3.1-184)$ & $1.6(0.3-2.7)$ & $4.5(1.4-13)$ & $<.001$ \\
\hline BSA $\left(\mathrm{m}^{2}\right)$ at repair & $0.4(0.23-1.9)$ & $0.27(0.17-0.39)$ & $0.37(0.24-0.78)$ & $<.001$ \\
\hline Male & $214(56 \%)$ & $26(62 \%)$ & $13(46 \%)$ & .15 \\
\hline Death & $1(1 \%)$ & $1(2 \%)$ & $1(4 \%)$ & .49 \\
\hline Baseline morphology & Mean (SD) or N (\%) & Mean (SD) or N (\%) & Mean (SD) or N (\%) & \\
\hline Diffuse RVOT hypoplasia & $252(56 \%)$ & $24(57 \%)$ & $18(64 \%)$ & .63 \\
\hline Right-sided aortic arch & $99(26 \%)$ & $12(29 \%)$ & $15(54 \%)$ & .05 \\
\hline Conal branch & $56(15 \%)$ & $11(26 \%)$ & $5(18 \%)$ & .56 \\
\hline PV atresia & $4(1 \%)$ & $1(2 \%)$ & $4(14 \%)$ & .15 \\
\hline PV (mm) & $7.1 \pm 2.7$ & $5.3 \pm 1.9$ & $4.2 \pm 2.4$ & .19 \\
\hline PV $z$ score & $-4 \pm 2.5$ & $-5 \pm 2.6$ & $-6.5 \pm 3.3$ & .30 \\
\hline Moderate or greater PI & $10(3 \%)$ & $7(16 \%)$ & $0(0 \%)$ & .023 \\
\hline $\mathrm{TV}(\mathrm{mm})$ & $14.2 \pm 0.4$ & $11.8 \pm 1.7$ & $11.4 \pm 2.4$ & .087 \\
\hline $\mathrm{TV} z$ score & $-2.5 \pm 1.5$ & $-2.1 \pm 1.5$ & $-2.5 \pm 1.5$ & .96 \\
\hline Minimum branch PA (mm) & $5.2 \pm 2.3$ & $5.0 \pm 2.9$ & $3.3 \pm 1.6$ & .003 \\
\hline Minimum branch PA $z$ score & $-1.5 \pm 2$ & $-2 \pm 4$ & $-3.6 \pm 2.9$ & .17 \\
\hline $\begin{array}{l}\text { Mean RVOT peak gradient (mm } \\
\mathrm{Hg} \text { ) }\end{array}$ & $70 \pm 26$ & $62 \pm 25$ & $58 \pm 23$ & .88 \\
\hline \multicolumn{5}{|l|}{ Operative summary } \\
\hline RVSP after initial intervention & $46 \pm 11$ & $44 \pm 9$ & $57 \pm 14$ & .058 \\
\hline $\mathrm{CPB}$ (min) & $113 \pm 43$ & $104 \pm 33$ & $146 \pm 59$ & .013 \\
\hline Ischemic time (min) & $72 \pm 24$ & $75 \pm 26$ & $88 \pm 22$ & .57 \\
\hline Transannular patch & $103(27 \%)$ & $16(38 \%)$ & $17(61 \%)$ & .088 \\
\hline Pulmonary valvotomy & $237(62 \%)$ & $16(38 \%)$ & $5(18 \%)$ & .11 \\
\hline Conduit & $12(3 \%)$ & $8(19 \%)$ & $2(7 \%)$ & .30 \\
\hline Final PV probe diameter $(\mathrm{mm})$ & $10.4 \pm 2$ & $9.6 \pm 1$ & $10.1 \pm 0.6$ & .051 \\
\hline Final RVSP (mm Hg) & $46 \pm 11$ & $44 \pm 9$ & $46 \pm 11$ & .46 \\
\hline Final gradient (mm Hg) & $20 \pm 9$ & $18 \pm 15$ & $14 \pm 5$ & .06 \\
\hline Surgical reoperations $\dagger$ & $29(8 \%)$ & $7(17 \%)$ & $3(11 \%)$ & $\mathrm{n} / \mathrm{a}$ \\
\hline Catheter reintervention $\dagger$ & $39(10 \%)$ & $11(26 \%)$ & $11(39 \%)$ & $\mathrm{n} / \mathrm{a}$ \\
\hline
\end{tabular}

IDEAL, Elective single-stage tetralogy of Fallot repair; EARLY, early primary surgical repair; $C A T H$, early transcatheter palliation; $B S A$, body surface area; $S D$, standard deviation; $R V O T$, right ventricular outflow tract; $P V$, pulmonary valve; $P I$, pulmonary insufficiency; $T V$, tricuspid valve; $P A$, pulmonary artery; $R V S P$, right ventricle systolic pressure; $C P B$, cardiopulmonary bypass; $n / a$, not available. $* P$ values are based on comparison of EARLY and CATH groups only. IDEAL group data are provided as a reference. $\dagger P$ values were not included because these are time-related outcomes; however, the absolute number and associated percentage for each group was included as a reference. 

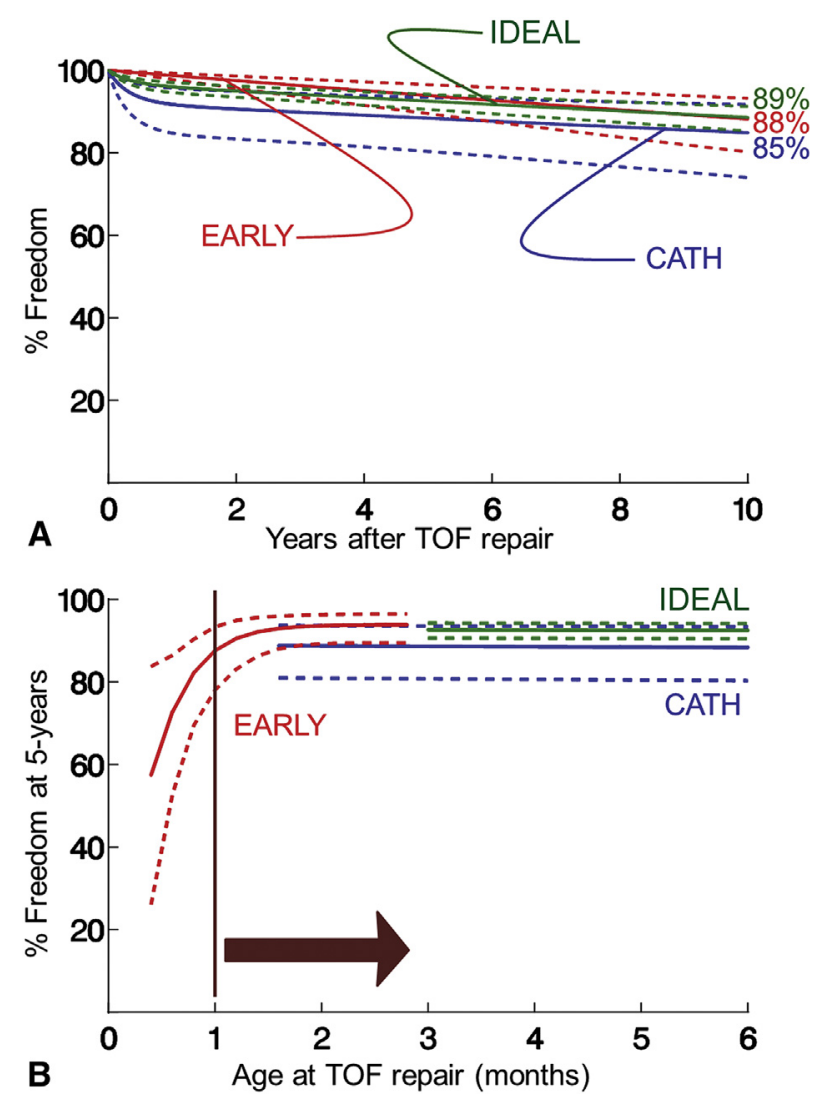

FIGURE 1. Risk-adjusted freedom from surgical reoperation (A) during overall follow-up and (B) as a nomogram depicting the 5-year reoperation relative to age at TOF repair. A, Risk-adjusted, 10-year freedom from any surgical reoperation for all 453 children stratified by timing of TOF repair. B, Nomogram depicting the impact of initial age of repair on the 5-year, risk-adjusted freedom from surgical reoperation for each respective group. Overall, the figures indicate that the timing of repair did not significantly affect the rate of reoperation except for neonates aged less than 1 month. Green curve: 383 children who underwent TOF repair at more than 3 months of age (IDEAL). Red curve: 42 children who underwent TOF repair at 3 months of less of age. Blue curve: 28 children who underwent transcatheter palliation before primary TOF repair (CATH). Solid lines are parametric estimates for the freedom from surgical reoperation enclosed by $68 \%$ confidence intervals (dashed lines). The estimated freedom from reoperation was based on mean or values for intraoperative needle right ventricular systolic pressure $(46 \mathrm{~mm} \mathrm{Hg})$, absent pulmonary valve (0.04), and diffuse right ventricular outflow tract stenosis (0.6) and age at operation (12.5 months). Note for the nomogram time is held constant at 5 years and age at operation remains variable. Each curve is truncated at minimum age of operation. IDEAL, Elective single-stage tetralogy of Fallot repair; EARLY, early primary surgical repair; $C A T H$, early transcatheter palliation; TOF, tetralogy of Fallot.

\section{Invasive Reintervention}

Surgical reoperation. Independently of time, 39 of 453 patients $(9 \%)$ underwent at least 1 surgical reoperation after primary TOF repair, with a resulting overall freedom from surgical reoperation of $83 \% \pm 2 \%$ at 10 years. The IDEAL, EARLY, and CATH groups underwent 29 of $383(8 \%), 7$ of
TABLE 2. Risk factors associated with increased surgical reoperation and nonsurgical catheter reintervention

\begin{tabular}{|c|c|c|}
\hline Variable & $\begin{array}{c}\text { Parameter } \\
\text { estimate (SE) }\end{array}$ & $\boldsymbol{P}$ \\
\hline \multicolumn{3}{|l|}{ Surgical reoperation } \\
\hline \multicolumn{3}{|l|}{ Early phase } \\
\hline $\begin{array}{l}\text { Younger age at primary } \\
\text { TOF repair (mo) }\end{array}$ & $0.02(0.006)$ & .0199 \\
\hline $\begin{array}{l}\text { Higher final needle } \\
\text { RVSP }(\mathrm{mm} \mathrm{Hg}) *\end{array}$ & $0.05(0.02)$ & .011 \\
\hline EARLY & $4.1(2.0)$ & .043 \\
\hline $\mathrm{CATH}_{\ddagger}$ & $0.92(0.82)$ & .26 \\
\hline $\begin{array}{l}\text { Interaction (strategy and } \\
\text { age at repair) } \dagger\end{array}$ & $-3.3(2.5)$ & .19 \\
\hline \multicolumn{3}{|l|}{ Constant phase } \\
\hline Absent pulmonary valve & $3.2(1.15)$ & .006 \\
\hline Diffuse RVOT stenosis & $2.3(1.0)$ & .027 \\
\hline EARLY $\ddagger$ & $.037(0.6)$ & .51 \\
\hline $\mathrm{CATH}_{\ddagger}$ & $-0.07(1.0)$ & .95 \\
\hline \multicolumn{3}{|c|}{ Nonsurgical catheter reintervention } \\
\hline $\begin{array}{l}\text { Smaller branch } \\
\text { pulmonary artery } z \\
\text { score }\end{array}$ & $-0.18(0.07)$ & .012 \\
\hline $\begin{array}{l}\text { Higher final needle } \\
\text { RVSP (mm Hg)* }\end{array}$ & $0.05(0.011)$ & $<.001$ \\
\hline EARLY & $0.98(0.5)$ & .038 \\
\hline CATH & $1.4(0.6)$ & .018 \\
\hline \multicolumn{3}{|l|}{ Constant phase } \\
\hline Absent pulmonary valve & $2.2(0.67)$ & $<.001$ \\
\hline $\begin{array}{l}\text { Smaller branch } \\
\text { pulmonary annulus } z \\
\text { score }\end{array}$ & $-0.25(0.12)$ & .037 \\
\hline EARLY $\ddagger$ & $-0.44(0.74)$ & .56 \\
\hline $\mathrm{CATH}_{\ddagger}$ & $1.2(1.2)$ & .33 \\
\hline
\end{tabular}

Incremental risk factors for the freedom from surgical reoperation and catheter reintervention. $S E$, Standard error; TOF, tetralogy of Fallot; $R V S P$, right ventricular systolic pressure; EARLY, early primary surgical repair; $C A T H$, early transcatheter palliation; RVOT, right ventricular outflow tract. *RVSP was measured at the end of the operation using needle manometer. $\dagger$ A significant interaction was associated with initial strategy and age at TOF repair, such that there was a disproportionate impact on the rate of surgical reoperation for very young infants, resulting an increased risk of reoperation if primary repair was undertaken at less than 1 month of age. $\ddagger$ Designates variables that were manually included in the final multivariable model to assess the risk of surgical reoperation and catheter reintervention among the IDEAL, EARLY, and CATH strategies. Parameter estimates were determined from multiphase risk-hazard analysis and represent the magnitude of the risk for reintervention. The PE for each variable is related to the hazard function and the polarity of the estimate (negative or positive) describes a decreased (negative) or increased (positive) association with each hazard phase (instantaneous risk of an event). In a multivariable hazard analysis, the overall influence on the hazard function is described by the total effect of each estimate in the model.

$42(17 \%)$, and 3 of $28(11 \%)$ reoperations, respectively. Overall, the 10-year freedom from reoperation was not significantly different among groups (Figure 1, A). However, the age-adjusted risk of reoperation was significantly increased in the EARLY group (Table 2). This difference was attributed to neonates who underwent primary TOF at less than 30 days (Figure 1, B). 
Catheter reintervention. Independently of time, $61 \mathrm{pa}-$ tients underwent at least 1 invasive catheter reintervention after primary TOF repair, resulting in an overall freedom from catheter reintervention of $76 \% \pm 3 \%$ at 10 years. There were 39, 11, and 11 catheter reinterventions in the IDEAL, EARLY, and CATH groups, respectively. The 10year freedom from reintervention was $80 \% \pm 3 \%$ for the IDEAL, $65 \% \pm 9 \%$ for the EARLY $(P<.11)$, and $31 \% \pm 14 \%$ for the CATH groups $(P<.002)$, corresponding to a significantly higher rate of reinterventions for the CATH group relative to the EARLY and IDEAL groups in an unadjusted comparison. When adjusting for additional important variables (Table 2), the EARLY $(76 \% \pm 6 \%$; $P=.038)$ and CATH $(53 \% \pm 16 \% ; P=.018)$ groups both demonstrated a decreased freedom from catheter reintervention versus the IDEAL ( $84 \% \pm 4 \%$ ) group, primarily attributed to reintervention soon $(<2$ years) after primary TOF repair (Figure 2, $A$ ).

The majority of catheter reinterventions were directed at the branch PAs $(\mathrm{n}=45)$. In an unadjusted evaluation, CATH $(53 \% ; P<.001)$ was associated with a decreased freedom from branch PA reintervention at 10 years compared with IDEAL repair $(87 \%)$, whereas EARLY $(76 \% ; P<.1)$ was not (Figure E2). Likewise, when risk adjusting, CATH remained a strong risk factor for branch PA reintervention (parameter estimate $[\mathrm{PE}]=1.1, P=.01$ ) versus EARLY repair $(\mathrm{PE}=0.66, P=.11$ ) (Figure $2, B)$. Of note, branch PA reintervention tended to occur early $(<2$ years) after TOF repair for the CATH and EARLY groups. A single patient in the EARLY group who underwent branch PA reintervention 8 years postrepair was the only one who received late branch PA reintervention among children in the EARLY and CATH groups. Baseline characteristics associated with branch PA reintervention included a smaller branch PA $z$ score $(\mathrm{PE}=-0.21, P<.001)$ and higher final intraoperative RVSP $(\mathrm{mm} \mathrm{Hg})$ measured on needle manometry $(\mathrm{PE}=0.04, P=.008)$

\section{Longitudinal Outcomes}

Somatic growth. Somatic growth, as estimated from repeated measurements of postoperative weight, increased at a nearly linear rate. When adjusting for the baseline preoperative weight, the 8-year rate of growth was not significantly different among the IDEAL, EARLY, and CATH groups (Figure 3). Preoperative weight was the most important predictor of postoperative growth $(P<.001)$.

Right ventricular systolic pressure. For the cohort overall, RVSP decreased in the early postoperative period from a mean of $41 \pm 1 \mathrm{~mm} \mathrm{Hg}$ immediately after surgery to $36 \pm 1 \mathrm{~mm} \mathrm{Hg}$ at 3 years before beginning to increase. At 8 years, RVSP was $38 \pm 2 \mathrm{~mm} \mathrm{Hg}$. The change in postoperative RVSP did not differ significantly for the EARLY $(P=.25)$ and $\mathrm{CATH}(P=.38)$ groups relative to the IDEAL
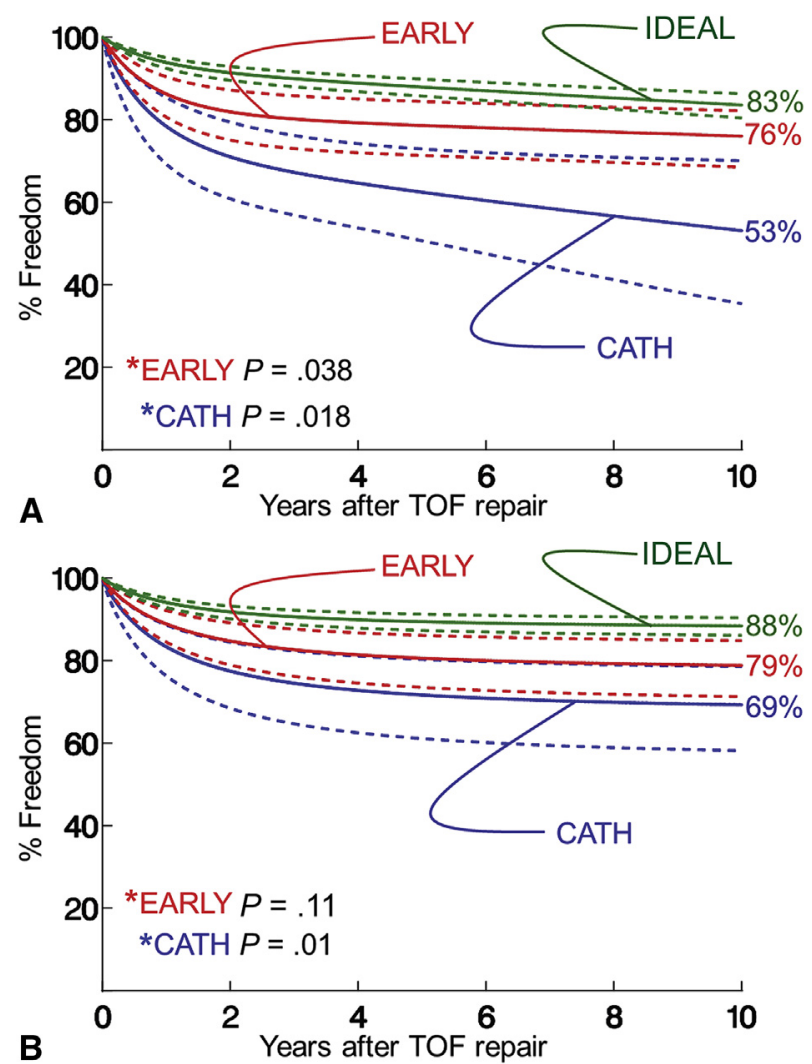

FIGURE 2. Freedom from (A) all catheter reinterventions and (B) branch pulmonary artery catheter reintervention. Risk-adjusted, 10-year freedom from catheter reinterventions for all 453 children stratified by timing of primary TOF repair for (A) all catheter reinterventions and (B) only catheter reinterventions directed at the branch pulmonary arteries. Both EARLY and CATH were independent risk factors for all catheter reinterventions. Alternatively, for branch pulmonary artery reintervention, CATH remained an independent risk factor, whereas EARLY did not. Green curve: 383 children who underwent TOF repair at more than 3 months of age (IDEAL). Red curve: 42 children who underwent TOF repair at 3 months or less of age (EARLY). Blue curve: 28 children who underwent transcatheter palliation before primary TOF repair (CATH). Solid lines are parametric estimates for the freedom from catheter reintervention enclosed by $68 \%$ confidence intervals (dashed lines). $* P$ values for the EARLY and CATH groups are compared with the IDEAL group. The estimated freedom from reoperation was based on mean or values for intraoperative needle right ventricular systolic pressure $(46 \mathrm{~mm} \mathrm{Hg}$ ), absent pulmonary valve (0.04), baseline branch pulmonary artery $z$ score $(-1.6)$, and baseline pulmonary valve annulus $z$ score $(-4)$. $P$ values obtained from multivariable risk-hazard analysis. $E A R L Y$, Early primary surgical repair; IDEAL, elective single-stage tetralogy of Fallot repair; $C A T H$, early transcatheter palliation; TOF, tetralogy of Fallot.

group (Figure 4). At 8 years, risk-adjusted RVSP was 38, 41, and $40 \mathrm{~mm} \mathrm{Hg}$ for the IDEAL, EARLY, and CATH groups, respectively.

Branch pulmonary artery growth. As might be expected in an unadjusted comparison, within the first year after TOF repair, branch PA size was significantly smaller for the EARLY group $(P=.02)$ and tended to be smaller for the 


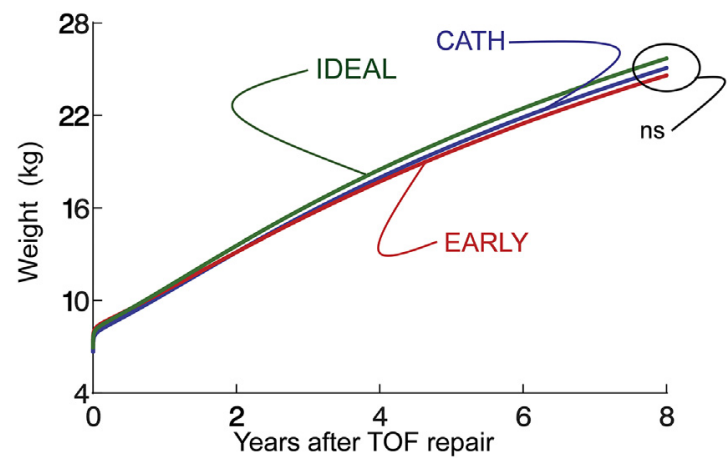

FIGURE 3. Somatic growth adjusted for weight at TOF repair. Somatic growth as measured by weight $(\mathrm{kg})$ after adjusting for weight at TOF repair stratified by IDEAL (green curve), EARLY (red curve), and CATH (blue curve) groups. When adjusted for preoperative weight $(P<.001)$ there was no difference in rate of growth among the groups. Each curve represents the weight-adjusted estimate of temporal trend for change in weight as evaluated by 1946 repeated measurements for 405 children. IDEAL, Elective single-stage tetralogy of Fallot repair; $C A T H$, early transcatheter palliation; $n s$, not significant; EARLY, early primary surgical repair; TOF, tetralogy of Fallot.

CATH group $(P=.09)$ when compared with the IDEAL group (Figure 5, $A$ ). Thereafter, branch PA growth for the EARLY group quickly caught up to the IDEAL group. Although the CATH group tended to have smaller branch PAs, long-term growth was not significantly different for the CATH $(P=.14)$ or EARLY $(P=.60)$ groups compared with IDEAL repair at 8 years post-TOF repair.

When risk adjusting for important variables, including baseline branch PA size, groups did not demonstrate a difference in branch PA growth soon after TOF repair (Table 3). EARLY repair tended to be associated with an increased rate of late branch PA growth (Figure 5, B). However, 8 years post-TOF, branch PA size was not significantly

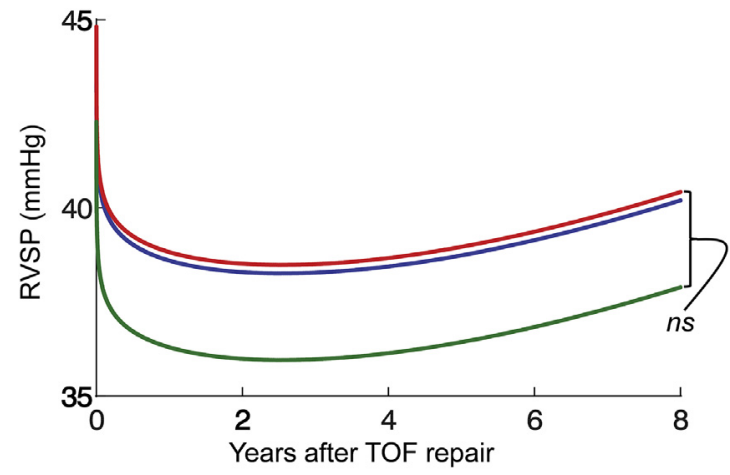

FIGURE 4. RVSP after TOF repair. Overall trends after TOF repair for RVSP stratified by IDEAL (green curve), EARLY (red curve), and CATH (blue curve) groups. There was no significant diffidence in the time-related change for RVSP among each group. Each curve represents the unadjusted estimate of temporal trend for change in RVSP as evaluated on 1106 echocardiogram reports for 324 children. $n s$, Not significant; $R V S P$, right ventricular systolic pressure; TOF, tetralogy of Fallot. different for EARLY $(P=.08)$ or CATH $(P=.40)$ when compared with IDEAL repair. Of note, although a younger age at the initial procedure was a risk factor for surgical reoperation, younger patients demonstrated an increased rate of branch PA growth postoperatively. This suggests that on the whole, branch PA growth displays an increased rate of growth for younger children.

Pulmonary valve insufficiency. For all patients, the prevalence of moderate or more (important) postoperative PI was $80 \% \pm 4 \% 8$ years after TOF repair. When adjusting for baseline morphologic characteristics, both the EARLY and CATH groups had an increased prevalence of PI relative to the IDEAL group (Table 4). When adjusting for pulmonary valvotomy (Table 4), with the remainder of the riskadjusted analysis kept the same, neither the EARLY nor the CATH group remained an independent factor for increased PI (Figure 6). Although a smaller percentage of patients in the EARLY $(38 \%)$ and CATH $(18 \% ; P=.11)$ groups underwent pulmonary valvotomy compared with IDEAL $(62 \%)$, these results suggest that, when possible, a valve-sparing operation for EARLY and CATH groups reduces the prevalence of important PI. It should be noted that baseline PI was not a significant predictor of postrepair PI.

\section{DISCUSSION}

For infants with severe TOF who require intervention early in life, the strategies of primary surgical repair and transcatheter palliation offer nearly equivalent long-term survival, somatic growth, RVSP, branch PA dimensions, and prevalence of important PI. Furthermore, these longterm outcomes are similar to children with TOF who undergo elective single-stage repair after 3 months of age (our institutional preference). However, intervention before this elective surgical window is associated with higher rates of invasive reintervention. That is, surgical repair during the neonatal period may come at a cost of higher rates of surgical reoperation, whereas early transcatheter palliation is associated with more frequent catheter reinterventions. Overall, long-term outcomes are excellent irrespective of management strategy.

\section{Initial Management}

For stable children with TOF, primary repair after 3 to 6 months of age is largely considered the ideal strategy. ${ }^{14-17}$ Alternatively, the management of infants with TOF who require early intervention, either secondary to cyanosis or prostaglandin dependence, still varies across western centers. $^{3,5,14}$ For example, both North American and European registries report a significant prevalence of SPS use for young children requiring staged palliation, ${ }^{14,18}$ as do various single-center reports. ${ }^{3,5}$ However, from approximately the mid-to-late 1990s, many large centers, including ours, have almost entirely abandoned SPS shunts as a bridge to complete repair. ${ }^{17,19}$ 

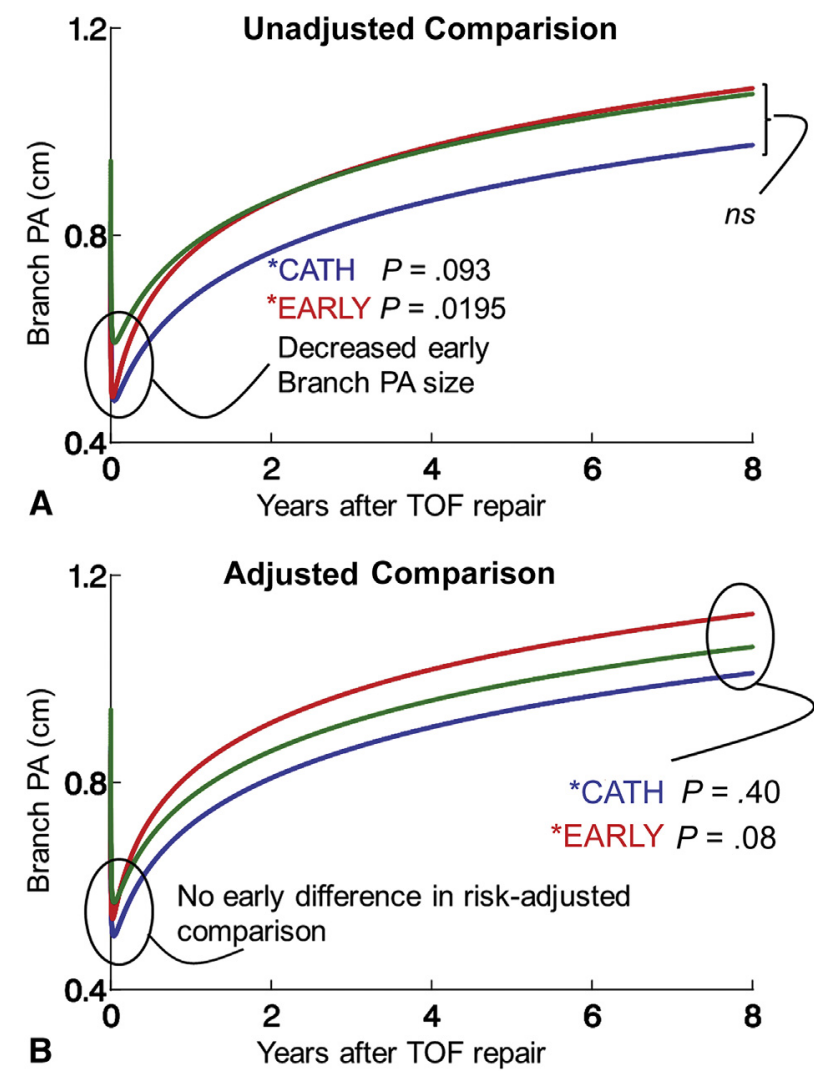

FIGURE 5. Branch PA growth after TOF repair. Unadjusted (A) and adjusted (B) branch PA growth after primary TOF repair stratified by IDEAL (green curve), EARLY (red curve), and CATH (blue curve) groups. The figures indicate that without adjusting for important factors, the EARLY and CATH groups demonstrated a decreased branch PA size relative to the IDEAL group. The EARLY group showed significant "catchup" growth and was equivalent to the IDEAL group, whereas the CATH group tended to have a slower rate of branch PA growth. However, when adjusting for important factors, including baseline branch PA size, there was no difference in initial branch PA size among the groups, and the EARLY group tended to demonstrate better late PA growth compared with both the IDEAL and CATH groups. Each curve represents the adjusted estimate of temporal trend for change in branch PA size $(\mathrm{cm})$ as evaluated on 1547 echocardiogram reports for 392 children. $* P$ values represent the comparison for (A) CATH and EARLY versus IDEAL and (B) EARLY and USUSAL versus CATH. Each variable in the risk-adjusted model, other than strategy, is held constant at the mean value: intraoperative needle right ventricular systolic pressure $(47 \mathrm{~mm} \mathrm{Hg})$, age at TOF repair (10 months), baseline branch pulmonary artery $z$ score $(-1.6)$, diffuse right ventricular outflow tract stenosis (0.6), and baseline pulmonary valve annulus $z$ score (-4). PA, Pulmonary artery; $n s$, not significant; $C A T H$, early transcatheter palliation; EARLY, early primary surgical repair; TOF, tetralogy of Fallot.

For children with severe cyanosis requiring earlier intervention, avoidance of SPS presents a dilemma (and a certain amount of clinical controversy). For these children, our institution favors early primary surgical repair or transcatheter palliation before complete surgical repair. Although risk factors to determine the initial treatment strategy are evaluated on a continuum, without strict guidelines for
TABLE 3. Characteristics associated with decreased branch pulmonary artery growth

\begin{tabular}{lcc}
\hline \multicolumn{1}{c}{ Variable } & $\begin{array}{c}\text { Parameter } \\
\text { estimate (SE) }\end{array}$ & $\boldsymbol{P}$ \\
\hline BPA growth & & \\
$\quad$ Overall phase & $1.3(0.39)$ & $<.001$ \\
$\quad$ Lower BSA $\left(\mathrm{m}^{2}\right)$ at & & \\
$\quad$ repair & $0.004(0.01)$ & .09 \\
$\quad$ Younger age (mo) at & & \\
$\quad$ repair & $-0.03(0.27)$ & .21 \\
Early phase & $0.19(0.22)$ & .36 \\
$\quad$ EARLY & $0.11(0.03)$ & $<.001$ \\
$\quad$ CATH & & \\
Smaller baseline branch & & .09 \\
$\quad$ PA $z$ score & $0.33(0.18)$ & .40 \\
Late phase & $0.13(0.15)$ & .068 \\
EARLY & $0.04(0.02)$ & \\
CATH & & .041 \\
Smaller baseline branch & & \\
$\quad$ PA $z$ score & $0.01(0.005)$ & \\
Higher intraoperative & & \\
$\quad$ RVSP (mm Hg)* & & \\
Diffuse RVOT & & \\
hypoplasia at baseline & & \\
\hline
\end{tabular}

Characteristics associated with decreased branch PA $(\mathrm{cm})$ growth after TOF repair. EARLY repair tented to demonstrate adequate catch-up branch PA growth as evident by the positive estimate and $P$ value that approaches significance. Characteristics are listed with $\mathrm{PE}, \nmid \mathrm{SE}$, and $P$ value comparison. $S E$, Standard error; $B P A$, branch pulmonary artery; $B S A$, body surface area; $E A R L Y$, early primary surgical repair; $C A T H$, early transcatheter palliation; $P A$, pulmonary artery; $R V S P$, right ventricular systolic pressure; $R V O T$, right ventricular outflow tract. *RVSP was measured at the end of the operation using needle manometer. $\dagger$ For mixed model regression, the PE is interpreted similar to that of a standard regression model. It represents the magnitude and polarity of the association between the independent variable and the dependent variable of interest (ie, size of branch pulmonary artery). For example, a PE of 0.33 for the EARLY group indicates that there tends to be an association with late pulmonary artery growth and EARLY surgical repair. The magnitude of the effect is related to the dimensional units of the risk factor.

dichotomization, our institution's general approach is to undertake primary surgical repair for infants considered to have a lower-risk profile. Conversely, if surgical risk is considered excessive, for example, end-organ dysfunction, low birth weight neonates, infection, and coexisting noncardiac malformation, we prefer transcatheter palliation.

The bias associated with this strategy inherently makes a straightforward comparison between groups difficult. Nevertheless, our findings provide important insight into the management of infants with TOF requiring early intervention. In this series, children who were managed with initial transcatheter palliation tended to be smaller and younger and harbor less favorable morphology than those who underwent early primary repair. Despite these differences, however, mortality with the transcatheter approach was favorable and similar to early primary repair.

\section{Risk of Reintervention}

Overall survival for young children after TOF is excellent. ${ }^{15}$ As such, the clinical cost associated with early 
TABLE 4. Characteristics associated an increased prevalence of pulmonary insufficiency

\begin{tabular}{lcc}
\hline \multicolumn{1}{c}{ Variable } & $\begin{array}{c}\text { Parameter } \\
\text { estimate (SE) }\end{array}$ & $\boldsymbol{P}$ \\
\hline PI without adjusting for valve-preserving operation & \\
EARLY & $1.3(0.60)$ & .028 \\
CATH & $2.2(0.77)$ & .004 \\
Smaller baseline branch PA & $-0.30(0.09)$ & $<.001$ \\
$\quad z$ score & $1.5(0.38)$ & \\
Diffuse RVOT stenosis* & $<.001$ \\
PI when adjusting for valve-preserving operation & \\
EARLY & $0.95(0.60)$ & .12 \\
CATH & $1.5(0.77)$ & .061 \\
Smaller baseline branch PA & $-0.33(0.09)$ & $<.001$ \\
$z$ score & & \\
Diffuse RVOT stenosis* & $1.5(0.38)$ & $<.001$ \\
Pulmonary valvotomy & $-1.6(0.37)$ & $<.001$ \\
\hline
\end{tabular}

Characteristics associated with an increased prevalence of important (moderate or greater) PI and the relative impact of a valve-preserving operation. Compared with the IDEAL group, both EARLY and CATH demonstrated a significantly increased prevalence of important PI when pulmonary valvotomy was not included in the risk-adjusted analysis. However, when including pulmonary valvotomy in the riskadjusted analysis, neither EARLY nor CATH remained significant risk factors for an increased prevalence of PI. This demonstrates that preserving the pulmonary valve is associated with a decreased prevalence of PI, irrespective of timing of the initial operation. Characteristics are listed with PEs, SE, and $P$ value. SE, Standard error; $P I$, pulmonary insufficiency; $E A R L Y$, early primary surgical repair; $C A T H$, early transcatheter palliation; $P A$, pulmonary artery; RVOT, right ventricular outflow tract. *Infundibular and valvular stenosis.

intervention for TOF is largely related to subsequent interventions. In our series, the risk of reoperation among strategies was similar. However, a caveat was observed for neonates who underwent primary repair before 1 month of age. For these children, there was a clear trend toward increased surgical reoperation. Our findings are corroborated by a recent report showing an increased risk of reintervention for children who underwent primary repair at less than 55 days of age. ${ }^{15}$ Cunningham and colleagues ${ }^{15}$ suggested that reintervention rates were increased among very young infants, in part because smaller branch PAs adversely affect blood flow across the right ventricular outflow tract, resulting in a diminished pulmonary valve growth potential. ${ }^{15}$ In our series, conduit use was higher for younger children, likely contributing to the nonlinear relationship between extremely young patients and disproportionally higher reoperation rates.

\section{Branch Pulmonary Artery Growth and Reintervention}

Hypoplasia, stenosis, and inadequate branch PA growth remain a significant long-term sequelae for patients with TOF. $^{20,21}$ In our series, as with a recent report comparing stenting with early repair, ${ }^{16}$ branch PA catheterization was the most frequent invasive reintervention. Likewise, both studies demonstrated that branch PA reintervention frequently occurred early after TOF repair, within 2 years of initial transcatheterization or early repair. It makes sense

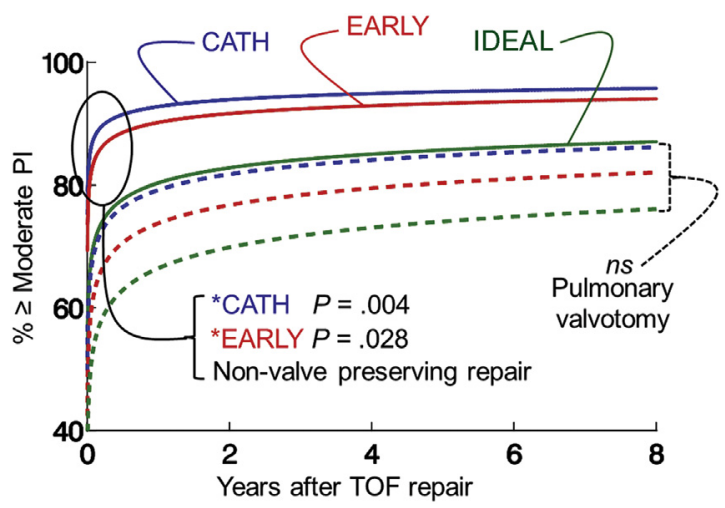

FIGURE 6. The relationship with valve-preserving operations and postoperative PI. Adjusted prevalence of moderate or greater PI after primary TOF repair for the IDEAL (green curve), EARLY (red curve), and CATH (blue curve) groups with further stratification by patients who underwent a pulmonary valvotomy (dashed curves) versus patients who underwent an nonvalved-preserving repair (solid curves). When comparing the adjusted prevalence of PI among each strategy (without taking valvepreserving operations into account), both *EARLY and *CATH were associated with a significant increase in PI relative to IDEAL. However, when further adjusting for pulmonary valvotomy repair, there was not a significant difference among each strategy. Each curve represents the adjusted estimate of temporal trend for prevalence of moderate or more PI as evaluated on 2246 echocardiogram reports for 443 children. $* P$ values represent the comparison for (A) EARLY and CATH versus IDEAL Each variable in the risk-adjusted model, other than strategy, is held constant at the mean value: baseline branch pulmonary artery $z$ score $(-1.6)$, diffuse right ventricular outflow tract stenosis (0.6). For the solid curves, the variable for pulmonary valvotomy was held at 0 , and for the dashed curves pulmonary valvotomy was kept at 1. CATH, Early transcatheter palliation; EARLY, early primary surgical repair; IDEAL, elective single-stage tetralogy of Fallot repair; $n s$, not significant; PI, pulmonary insufficiency; TOF, tetralogy of Fallot.

that very young patients undergoing transcatheter intervention or early repair would be predisposed to early branch PA reintervention. However, it seems reassuring that in the long term, rates of branch PA reintervention decreased among these children. Furthermore, the risk-adjusted follow-up suggests that such children, on average, do not develop statistically significant decrements in branch PA dimensions. Correspondingly, long-term trends in RVSP remained within reasonable limits for children managed by early primary surgical repair or transcatheter palliation.

\section{Somatic Growth and Pulmonary Insufficiency}

Our results did not demonstrate a significant difference in the long-term growth among either strategy. When adjusting for the preoperative weight, children who underwent early primary surgical repair or early transcatheter palliation displayed growth comparable to that of children who underwent primary repair at the ideal age. These results are similar to a recent comparison that demonstrated adequate catch-up growth after primary repair during the neonatal period when compared with patients who underwent a full repair later in life. ${ }^{2}$ 
PI has been a well-documented and deleterious sequel after TOF repair. ${ }^{22-24}$ As such, it is not surprising that a large proportion of patients in our study ultimately developed moderate or worse PI. Although our initial risk-adjusted analysis found a significant increase in the prevalence of PI for both early surgical repair and early transcatheter intervention, neither strategy maintained an independent association with PI when subsequently adjusting for pulmonary valve-preserving operation (ie, pulmonary valvotomy). In terms of limiting PI, therefore, the implication is that preservation of the pulmonary valve is more important than the timing of the initial intervention, a finding consistent with multiple previous studies. ${ }^{23-25}$

\section{Study Limitations}

We acknowledge limitations in a retrospective analysis of this kind. Clinical decisions were inherently biased because of preferred institutional practice patterns. This bias makes comparisons difficult, but it is for this reason that we have used advanced techniques for risk adjustment to try and account for patient-specific differences. The small patient numbers hamper the fidelity of risk adjustment. A randomized trial would be the ideal approach for an unbiased comparison of the 2 clinical strategies but would be difficult to implement and seems unlikely. Finally, we have focused on a small number of clinical end points. It is possible that important differences in noncardiac morbidity, recovery times, or cost exist between the 2 strategies that we were not able to explore in our analysis.

\section{CONCLUSIONS}

For infants with severe TOF who require intervention early in life, primary surgical repair and transcatheter palliation offer nearly equivalent long-term survival, somatic growth, RVSP branch PA dimensions, and prevalence of PI. Early transcatheter palliation comes at a cost of more frequent catheter reinterventions, whereas neonatal surgical repair tends to be associated with increased rates of surgical reoperation. Overall, long-term outcomes are excellent irrespective of management strategy.

\section{Conflict of Interest Statement}

Authors have nothing to disclose with regard to commercial support.

\section{References}

1. Walsh EP, Rockenmacher S, Keane JF, Hougen TJ, Lock JE, Castaneda AR. Late results in patients with tetralogy of Fallot repaired during infancy. Circulation. 1988;77:1062-7.

2. Woldu KL, Arya B, Bacha EA, Williams IA. Impact of neonatal versus nonneonatal total repair of tetralogy of Fallot on growth in the first year of life. Ann Thorac Surg. 2014;98:1399-404.

3. Kanter KR, Kogon BE, Kirshbom PM, Carlock PR. Symptomatic neonatal tetralogy of Fallot: repair or shunt? Ann Thorac Surg. 2010;89:858-63.
4. Karl TR, Sano S, Pornviliwan S, Mee RB. Tetralogy of Fallot: favorable outcome of nonneonatal transatrial, transpulmonary repair. Ann Thorac Surg. 1992;54:903-7.

5. Fraser CD Jr, McKenzie ED, Cooley DA. Tetralogy of Fallot: surgical management individualized to the patient. Ann Thorac Surg. 2001;71:1556-63.

6. Pigula FA, Khalil PN, Mayer JE, del Nido PJ, Jonas RA. Repair of tetralogy of Fallot in neonates and young infants. Circulation. 1999;100:II157-61.

7. Daubeney PE, Blackstone EH, Weintraub RG, Slavik Z, Scanlon J, Webber SA. Relationship of the dimension of cardiac structures to body size: an echocardiographic study in normal infants and children. Cardiol Young. 1999; 9:402-10.

8. Blackstone EH, Naftel DC, Turner ME Jr. The decomposition of time-varying hazard into phases, each incorporating a separate stream of concomitant information. J Am Stat Assoc. 1986;615-24.

9. Breiman L. Bagging predictors. Mach Learn. 1996;24:123-40.

10. Rohde L, Zhi G, Aranki SF, Beckel NE, Lee RT, Reimold SC. Gender associated differences in left ventricular geometry in patients with aortic valve disease and effect of distinct overload subsets. Am J Cardiol. 1997;80:475-80.

11. Rajeswaran J, Blackstone EH. A multiphase non-linear mixed effects model: an application to spirometry after lung transplantation. Stat Methods Med Res. 2017; 26:21-42.

12. Rajeswaran J, Blackstone EH, Ehrlinger J, Li L, Ishwaran H, Parides MK. Probability of atrial fibrillation after ablation: using a parametric nonlinear temporal decomposition mixed effects model. Stat Methods Med Res. January 5, 2016 [Epub ahead of print].

13. Beach JM, Mihaljevic T, Rajeswaran J, Marwick T, Edwards ST, Nowicki ER, et al. Ventricular hypertrophy and left atrial dilatation persist and are associated with reduced survival after valve replacement for aortic stenosis. J Thorac Cardiovasc Surg. 2014;147:362-9.e8.

14. Al Habib HF, Jacobs JP, Mavroudis C, Tchervenkov CI, O'Brien SM, Mohammadi S, et al. Contemporary patterns of management of tetralogy of Fallot: data from the Society of Thoracic Surgeons Database. Ann Thorac Surg. 2010;90:813-20.

15. Cunningham ME, Donofrio MT, Peer SM, Zurakowski D, Jonas RA, Sinha P. Optimal timing for elective early primary repair of tetralogy of Fallot: analysis of intermediate term outcomes. Ann Thorac Surg. 2017;103:845-52.

16. Sandoval JP, Chaturvedi RR, Benson L, Morgan G, Van Arsdell G, Honjo O, et al. Right ventricular outflow tract stenting in tetralogy of Fallot infants with risk factors for early primary repair. Circ Cardiovasc Interv. 2016;9.

17. Van Arsdell GS, Maharaj GS, Tom J, Rao VK, Coles JG, Freedom RM, et al. What is the optimal age for repair of tetralogy of Fallot? Circulation. 2000; 102:III123-9.

18. Jacobs JP, Jacobs ML, Maruszewski B, Lacour-Gayet FG, Clarke DR, Tchervenkov CI, et al. Current status of the European Association for CardioThoracic Surgery and the Society of Thoracic Surgeons Congenital Heart Surgery Database. Ann Thorac Surg. 2005;80:2278-84.

19. Najm HK, Van Arsdell GS, Watzka S, Hornberger L, Coles JG, Williams WG. Primary repair is superior to initial palliation in children with atrioventricular septal defect and tetralogy of Fallot. J Thorac Cardiovasc Surg. 1998;116:905-13.

20. Di Donato RM, Jonas RA, Lang P, Rome JJ, Mayer JE Jr, Castaneda AR. Neonatal repair of tetralogy of Fallot with and without pulmonary atresia. J Thorac Cardiovasc Surg. 1991;101:126-37.

21. Reddy VM, Liddicoat JR, McElhinney DB, Brook MM, Stanger P, Hanley FL. Routine primary repair of tetralogy of Fallot in neonates and infants less than three months of age. Ann Thorac Surg. 1995;60:S592-6.

22. Ho KW, Tan RS, Wong KY, Tan TH, Shankar S, Tan JL. Late complications following tetralogy of Fallot repair: the need for long-term follow-up. Ann Acad Med Singapore. 2007;36:947-53.

23. Vida VL, Angelini A, Guariento A, Frescura C, Fedrigo M, Padalino M, et al. Preserving the pulmonary valve during early repair of tetralogy of Fallot: anatomic substrates and surgical strategies. J Thorac Cardiovasc Surg. 2015; 149:1358-63.e1.

24. Borowski A, Ghodsizad A, Litmathe J, Lawrenz W, Schmidt KG, Gams E. Severe pulmonary regurgitation late after total repair of tetralogy of Fallot: surgical considerations. Pediatr Cardiol. 2004:25:466-71.

25. Vida VL, Guariento A, Castaldi B, Sambugaro M, Padalino MA, Milanesi O, et al. Evolving strategies for preserving the pulmonary valve during early repair of tetralogy of Fallot: mid-term results. J Thorac Cardiovasc Surg. 2014;147:687-96.

Key Words: catheter intervention, congenital heart disease, neonatal repair, tetralogy of Fallot 


\section{APPENDIX E1. VARIABLE SELECTION}

Parametric hazard analysis and longitudinal mixed-model analysis are regression techniques that fit a "baseline" parametric model to the available data using a mathematical equation. Similar to other regression techniques (ie, linear and logistic regression), selected patient-specific and procedural characteristics, including demographic, functional, morphologic, and surgical factors, can be incorporated into these models to identify if a significant relationship exists with an independent variable and the outcome variable of interest. Although there are nuanced technical differences in developing the final multivariable (risk-adjusted) models, in general, variable selection for hazard models and longitudinal mixed-models is conducted in a similar fashion.

Selected patient-specific and procedural characteristics were extracted from clinical reports. After initial screening, as previously described, ${ }^{\mathrm{E} 1}$ common transformations of continuous variables were examined to match the scale of the variable with the scale of the risk (calibration). To minimize the risk of model overdetermination, ordinal variables with less than 5 associated events were excluded. Missing values for baseline (independent) variables were estimated with 5-fold multiple imputation via the Markov Chain Monte Carlo technique (SAS PROC MI; SAS Institute Inc, Cary, $\mathrm{NC})^{\mathrm{E} 2}$; however, outcome (dependent) variables of interest were not imputed. To test the robustness and "generalizability" of a variable to a wider study population, variable selection was guided by bootstrap resampling (bagging). Variables appearing in 50\% or more of models or clusters were considered reliable for inclusion.

For hazard analysis, bootstrap-proven variables were entered into each model in a semidirected (stepwise) manner, with initial entry criteria for variable entry set at the $P$ value of .15 or less and final selection criteria set at the $P$ value of .05 or less level of significance (longitudinal regression analysis does not allow for an automated selection process, described later). Subsequently, various interaction terms were examined for additional entry into the final multivariable model. Finally, a $P$ value of less than .05 was considered significant; however, important variables of interest were sometimes included with a $P$ value greater than .05 if they were of clinical interest (ie, procedure type or baseline somatic measurements) or important for model adjustment (ie, interaction terms).

Although statistical rules for incorporation of an interaction term are somewhat ambiguous, our practice is based on the spirit of the rule, that if it can be reasonably demonstrated that 2 variables demonstrate a conditional effect on one another, there is rationale for including an interaction term. Interaction terms were created between 2 variables in the final model that were perceived to be related on the basis of clinical reasoning. If parameter estimates and significance of either independent variable used to generate the interaction term were largely affected by the interaction term, it was assumed there was a conditional effect between these variables. In this case, the interaction term was included in the final model. In addition, interaction terms were graphically evaluated to identify their influence on the response variable.

\section{LONGITUDINAL (REPEATED-MEASURES) DATA ANALYSIS}

The benefits, rational, and basic methodological strategy of mixed models have been published. ${ }^{\mathrm{E} 3, \mathrm{E} 4}$ In the current analysis, nonlinear mixed-model regression analysis was used to characterize the postoperative temporal trends for somatic growth, branch pulmonary artery growth, right ventricular systolic pressure, and pulmonary insufficiency (PI) via repeated measurements (SAS PROC NLMIXED), using a multiphase parametric technique. ${ }^{\text {E4-E6 }}$ The severity of PI was extracted from echocardiogram reports. Initially, the amount of PI was classified on the basis of a 7-point categoric scale ranging from no insufficiency to severe insufficiency. For nonlinear mixed model analysis, PI was dichotomized by the presence of moderate or worse disease (moderate or greater). As such, results for PI represent the prevalence of moderate or greater (important) PI over time.

Unlike hazard analysis, PROC NLMIXED does not have built-in covariable selection algorithms. Variable selection is achieved first by forcing bootstrap-proven variables into each phase to determine their effect. If it is determined to be important for an individual phase, the variable is selected for multivariable analysis. If it is selected in all phases of the model, and the parameter estimates are approximately equal, the variable is used for the "overall" phase-meaning it has the same magnitude of influence and similar level of significance on the dependent variable throughout the time period of interest. This process is repeated until all variables have been considered. Variables with a $P$ value less than .1 on initial screening are entered into the model for final selection. Variables with a $P$ value of .05 or less are retained in the final multivariable model. ${ }^{\mathrm{E} 3}$ Additional variables may be included in the final model if deemed clinically important (ie, baseline age or branch pulmonary artery size) or are relevant for the specific analysis (ie, procedure type).

For nonlinear mixed models, parameters of the variance covariance matrix are estimated via the method of maximum likelihood estimation and computed using the Hessian matrix. Maximum likelihood estimates are obtained using the marginal likelihood function, which are calculated using the Gauss-Hermite quadrature, an "exact" method of integration. Final model selection is guided by the corrected Akaike's information criteria and concordance correlation coefficient $\left(r_{c}\right)$ between observed and predicted values. ${ }^{\mathrm{E} 4 \mathrm{E} 6}$ 


\section{E-References}

E1. Daubeney PE, Blackstone EH, Weintraub RG, Slavik Z, Scanlon J, Webber SA. Relationship of the dimension of cardiac structures to body size: an echocardiographic study in normal infants and children. Cardiol Young. 1999;9:402-10.

E2. Rubin DB. Multiple Imputation for Nonresponse in Surveys. New York, NY: John Wiley \& Sons; 2004.

E3. Beach JM, Mihaljevic T, Rajeswaran J, Marwick T, Edwards ST, Nowicki ER, et al. Ventricular hypertrophy and left atrial dilatation persist and are associated with reduced survival after valve replacement for aortic stenosis. J Thorac Cardiovasc Surg. 2014;147:362-9.e8.
E4. Rajeswaran J, Blackstone EH. A multiphase non-linear mixed effects model: an application to spirometry after lung transplantation. Stat Methods Med Res. 2017;26:21-42.

E5. Rohde L, Zhi G, Aranki SF, Beckel NE, Lee RT, Reimold SC. Gender associated differences in left ventricular geometry in patients with aortic valve disease and effect of distinct overload subsets. Am J Cardiol. 1997;80:475-80.

E6. Rajeswaran J, Blackstone EH, Ehrlinger J, Li L, Ishwaran H, Parides MK. Probability of atrial fibrillation after ablation: using a parametric nonlinear temporal decomposition mixed effects model. Stat Methods Med Res. January 5, 2016 [Epub ahead of print].

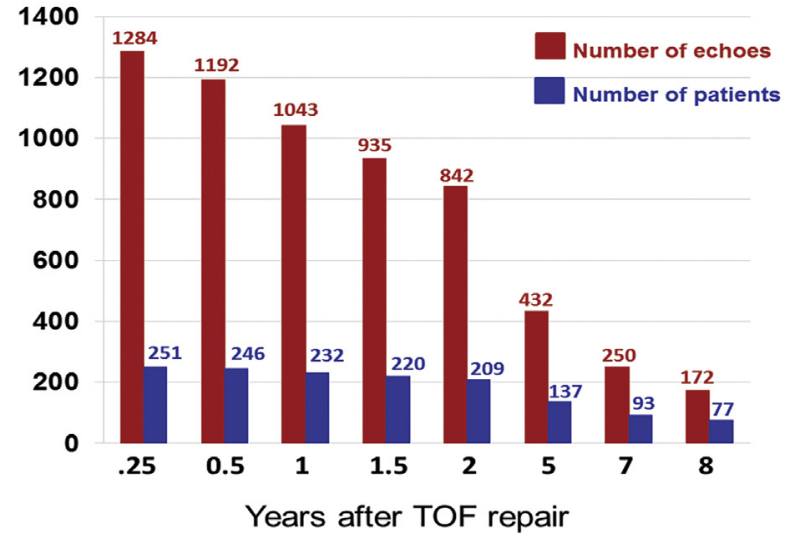

FIGURE E1. Number of patients with postoperative echocardiograms available. Number of patients with postoperative echocardiograms available at and beyond various measurement points with the number of echocardiograms available for analysis at each point. Note the time intervals are not uniform. TOF, Tetralogy of Fallot.

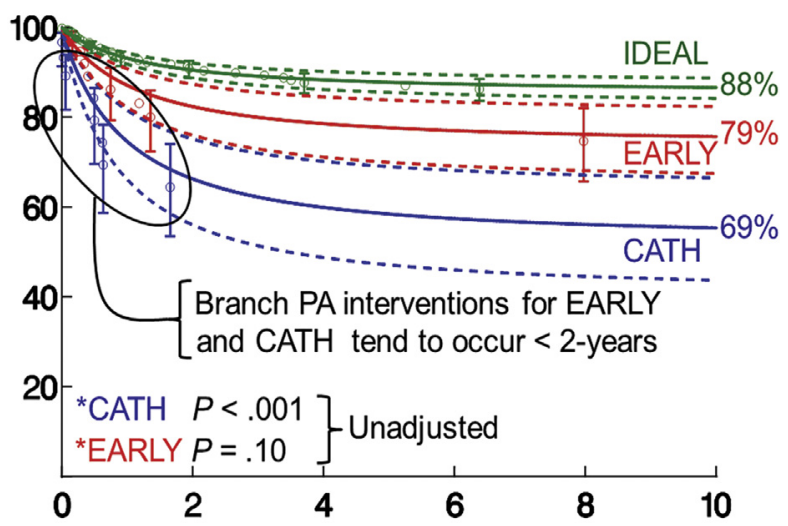

FIGURE E2. Unadjusted freedom from branch pulmonary artery $(P A)$ catheter reintervention. Unadjusted 10-year freedom from branch PA catheter reinterventions for all 453 children stratified by timing of primary tetralogy of Fallot (TOF) repair. For EARLY and CATH groups, branch PA reintervention occurred relatively soon after TOF repair; all but 1 reintervention occurred after 2 years. Green curve: 383 children who underwent TOF repair at more than 3 months (IDEAL). Red curve: 42 children who underwent TOF repair at 3 months or less (EARLY). Blue curve: 28 children who underwent transcatheter palliation before primary TOF repair (CATH). Each circle represents a reintervention positioned on the vertical axis by the Kaplan-Meier estimator accompanied by confidence limits (vertical bars) equivalent to \pm 1 standard error. Solid lines are parametric estimates for the freedom from catheter reintervention enclosed by $68 \%$ confidence intervals (dashed lines). $* P$ values for the EARLY and CATH groups are compared with the IDEAL group. CATH, Early transcatheter palliation; EARLY, early primary surgical repair; IDEAL, elective single-stage tetralogy of Fallot. 